\title{
Prevalência de morbidade hospitalar por doenças crônicas não transmissíveis em Salvador (BA): dados DATASUS
}

\author{
Prevalence of hospital morbidity due to chronic non-communicable diseases in \\ Salvador (BA): Datasus data
}

Jamile das Virgens Silva ${ }^{1}$, Fábio Rodrigo Santana dos Santos², Edilene Maria Queiroz Araújo ${ }^{3 *}$

\begin{abstract}
${ }^{1}$ Nutricionista pela União Metropolitana de Educação e Cultura (UNIME), Especialista em Nutrição Clínica Funcional pela Universidade Cruzeiro do Sul (UNISUL), Mestranda do Programa de Pós-graduação Processos Interativos dos Órgãos e Sistemas da UFBA; ${ }^{2}$ Bacharel em Nutrição pela UNEB, Mestre em Alimentos Nutrição e Saúde pela UFBA, Professor Assistente da Universidade do Estado da Bahia (UNEB); ${ }^{3}$ Nutricionista pela UNEB, Doutora em Biotecnologia pela Universidade Estadual de Feira de Santana (UEFS), Professora Adjunto da UNEB e do Programa de Pós-graduação Processos Interativos dos Órgãos e Sistemas da UFBA
\end{abstract}

\begin{abstract}
Resumo
Introdução: as doenças crônicas não transmissíveis (DCNT) são consideradas como um dos maiores problemas globais de saúde pública da atualidade. Entre elas, podem-se destacar o diabetes mellitus (DM), a hipertensão arterial sistêmica (HAS) e a obesidade. As DCNT causam agravos à saúde e altas taxas de internação e óbitos, quando observadas isoladamente. Além disso, quando associadas, tendem a elevar o grau de morbidade, causando grandes prejuízos aos cofres públicos. Objetivo: Verificar a prevalência de internações e óbitos por DM, HAS e obesidade na população de Salvador. Metodologia: trata-se de um estudo descritivo, com base em dados do Departamento de Informação e Informática do SUS (DATASUS), sobre internações e óbitos ocorridos no período de janeiro a novembro de 2019, no município de Salvador (BA). A coleta dos dados eletrônicos foi realizada no mês de janeiro de 2020 e eles foram apresentados em forma de prevalência. Resultado: das internações registradas em Salvador, 0,63\% foram associadas a DM, $0,03 \%$ à obesidade e $0,85 \%$ a HAS como causa principal. Em relação aos óbitos, $0,80 \%$ foram associados a DM e $0,56 \%$ a HAS. Não foram registrados óbitos associados à obesidade como causa principal, nesse período. Conclusão: houve maior prevalência de mulheres e idosos nas internações e óbitos por HAS e DM. O uso dos dados do DATASUS foi de extrema importância para a identificação do perfil de morbidade hospitalar. No entanto, a fim de garantir a consistência e a qualidade dos dados, é necessária uma padronização no modo de coleta.
\end{abstract}

Palavras-chave: Hipertensão. Diabetes. Obesidade. Internações. Óbitos. Morbidade hospitalar.

\begin{abstract}
Introduction: chronic non-communicable diseases (NCDs) are considered to be one of the biggest global public health problems today. Among them, diabetes mellitus (DM), systemic arterial hypertension (SAH) and obesity can be highlighted. NCDs cause health problems and high rates of hospitalization and deaths, when observed in isolation. In addition, when associated, they tend to increase the degree of morbidity, causing great damage to public coffers. Objective: to verify the prevalence of hospitalizations and deaths due to DM, SAH and obesity in the population of Salvador. Methods: this is a descriptive study, based on data from the Department of Information and Informatics of SUS (DATASUS), on hospitalizations and deaths from January to November 2019, in the city of Salvador (BA). The collection of electronic data was carried out in January 2020 and they were presented as prevalence. Results - Of the hospitalizations registered in Salvador, $0.63 \%$ were associated with DM, $0.03 \%$ with obesity and $0.85 \%$ with SAH as the main cause. Regarding deaths, $0.80 \%$ was associated with DM and $0.56 \%$ with SAH. There were no deaths associated with obesity as the main cause during this period. Conclusion: there was a higher prevalence of women and the elderly in hospitalizations and deaths due to SAH and DM. The use of DATASUS data was extremely important for the identification of the hospital morbidity profile. However, in order to guarantee the consistency and quality of the data, it is necessary to standardize the mode of collection. Keywords: Hypertension. Diabetes. Obesity; Hospitalizations. Deaths. Hospital Morbidity.
\end{abstract}

\section{INTRODUÇÃO}

As doenças crônicas não transmissíveis (DCNT) foram responsáveis por cerca de $56,9 \%$ das mortes no Brasil no ano de 2017, na faixa etária de 30 a 69 anos, e são

Correspondente/Corresponding: *Edilene Maria Queiroz Araújo - End; Rua Embira, n.149, Ed.Paládio, apto.1204, Paramares - Tel: (71)999772922. consideradas como um dos maiores problemas globais de saúde pública da atualidade (MINISTÉRIO DA SAÚDE, 2019). Entre as DCNT, podem-se destacar o Diabetes Mellitus (DM), a Hipertensão Arterial Sistêmica (HAS) e a obesidade, que associadas a outros fatores (obesidade abdominal, hipertrigliceridemia e HDL baixo), compõem a Síndrome Metabólica (SM) que, consequentemente, 
se caracteriza como outro grande problema de saúde pública, causando grandes prejuízos aos cofres públicos (ARAÚJO, 2016).

Define-se DM como um distúrbio metabólico caracterizado por hiperglicemia persistente, decorrente de deficiência na produção de insulina ou na sua ação, ou em ambos os mecanismos, ocasionando complicações em longo prazo. Em 2017, a Federação Internacional de Diabetes (International Diabetes Federation) estimou que $8,8 \%$ da população mundial, com 20 a 79 anos de idade $(424,9$ milhões de pessoas), viviam com diabetes. Se as tendências atuais persistirem, o número de pessoas com diabetes será superior a 628,6 milhões em 2045 (SBD, 2019). Segundo estimativa, até 2030 , o DM pode saltar de nona para sétima causa mais importante de morte em todo o mundo (FLOR, CAMPOS, 2017). Indivíduos com diabetes apresentam maiores taxas de hospitalização, em comparação com os que não têm diabetes, além de maior duração da hospitalização. Além disso, DM e suas complicações constituem as principais causas de mortalidade precoce na maioria dos países, pois aproximadamente 4 milhões de pessoas com idade entre 20 e 79 anos morreram por diabetes em 2015, o equivalente a um óbito a cada 8 segundos (SBD, 2019). Frequentemente, a DM está associada a outras comorbidades, como a HAS, dislipidemia, estado pró-trombótico e disfunção cardíaca autônoma. Estudos mostram que a prevalência de hipertensão é aproximadamente o dobro entre os diabéticos, em comparação com os não diabéticos, e o risco de doença cardiovascular é cerca de quatro vezes maior em pacientes com ambas as doenças (FRANCISCO et al., 2018).

A HAS é condição clínica multifatorial, caracterizada por elevação sustentada dos níveis pressóricos $\geq 140$ e/ ou $90 \mathrm{mmHg}$ (sistólica e diastólica, respectivamente). No Brasil, a HAS atinge 32,5\% (36 milhões) de indivíduos adultos, mais de $60 \%$ dos idosos, contribuindo, direta ou indiretamente, para $50 \%$ das mortes por doença cardiovascular (SBC, 2017). Importante é destacar que, a evolução tecnológica e o trabalho assalariado contribuem para o aumento do sedentarismo, o consumo de alimentos ultraprocessados, a maior ingestão de sal, a dislipidemia, o sobrepeso e a obesidade, que se destacam como fatores de risco comprovadamente associados à hipertensão arterial (DANTAS et al., 2018). Além disso, estudos mostram que outras comorbidades associadas podem aumentar o risco de hipertensão arterial, como a obesidade (FERREIRA, SZWARCWALD, DAMACENA, 2019; SANTIAGO et al., 2019), e essa condição comumente é vista como relacionada à HAS, como é mostrado por Dutra et al., num estudo realizado em 2019, que avaliou a prevalência de HAS e a obesidade numa região do Distrito Federal, onde $25,4 \%$ da amostra apresentou HAS e excesso de peso concomitantemente, e o teste qui-quadrado mostrou associação significativa entre HAS e excesso de peso $(p=0,001)$ (DUTRA et al., 2019).

A obesidade é uma doença de etiologia complexa e multifatorial, resultante da interação de genes, ambien- te, estilos de vida e fatores emocionais (ABESO, 2016), podendo ser definida pelo grau de armazenamento de gordura no organismo, associado a riscos para a saúde, devido à sua relação com várias complicações metabólicas (ARAÚJO et al., 2019). Segundo Dias et al. (2017), para a Organização Mundial de Saúde (OMS), a obesidade é definida como condição crônica caracterizada pelo acúmulo excessivo de gordura, o que traz repercussões para a saúde, e, por isso, é categorizada, na Classificação Estatística Internacional de Doenças e Problemas Relacionados à Saúde, versão 10 (CID-10). No Brasil, diferentes documentos governamentais seguem a definição da OMS, categorizando a obesidade, simultaneamente, como doença e como fator de risco para outras doenças, como condição crônica multifatorial complexa e, ainda, como manifestação de insegurança alimentar e nutricional (DIAS et al., 2017). A obesidade tem sido historicamente considerada de forma isolada de outros grandes desafios globais. Por isso, o relatório da Comissão The Lancet, publicado em 2019, colocou a obesidade num contexto mais amplo, o da sindemia global de obesidade, desnutrição e mudanças climáticas. Uma sindemia é a "presença de dois ou mais estados de doença que interagem de forma adversa, afetando negativamente o curso mútuo de cada trajetória da doença, aumentando a vulnerabilidade e tornando-se mais deletérios por iniquidades experimentadas" (SWINBURN et al., 2019). A obesidade cresceu de 11,8\%, em 2006, para 19,9\%, em 2016 (ARAÚJO et al. 2019). Estima-se que, atualmente, no mundo, cerca de 475 milhões de adultos sejam obesos, projetando-se números ainda mais impressionantes para a próxima década (MALTA et al., 2014).

Assim, considerando os agravos à saúde causados pelas DCNT e sua malignidade quando associadas, como na SM, este estudo teve como objetivo verificar a prevalência de internações e óbitos por DM, HAS e obesidade na população de Salvador (BA).

\section{METODOLOGIA}

Trata-se de um estudo descritivo, com base em dados secundários, sobre a morbidade hospitalar ocorrida no período de janeiro a novembro de 2019 (não foi possível coletar dados do mês de dezembro, pois no momento da consulta, eles não estavam disponíveis). Os dados foram obtidos no Sistema de Informação Hospitalar do Sistema Único de Saúde (SIH/SUS), que pertence à base de dados do Departamento de Informação e Informática do SUS (DATASUS), plataforma de Informações de Saúde (TABNET) - tabulador de dados (http://www2.datasus. gov.br/DATASUS/index.php).

Foram consideradas as variáveis: sexo (masculino e feminino), idade (adulto: de 20 a 59 anos; e idoso: a partir de 60 anos), cor ou raça (branca, preta, parda, amarela, indígena, sem informação). A amostra foi composta por dados referentes a internações e óbitos no município de Salvador (BA), pesquisando-se a causa segundo a lista 
de morbidades da CID10 (http://tabnet.datasus.gov.br/ $\mathrm{cgi} / \mathrm{sih} / \mathrm{mxcid} 10 \mathrm{~lm} . \mathrm{htm})$, com o uso dos códigos E10-E14 para diabetes mellitus, 110 para hipertensão essencial (primária), e E66 para obesidade.

A coleta dos dados eletrônicos foi realizada no mês de janeiro de 2020. Eles foram inseridos em planilhas do programa Microsoft Office Excel - 2010 e foram apresentados em forma de prevalência.

\section{RESULTADOS}

Segundo dados do DATASUS, de janeiro a novembro de 2019, foram registradas 586.453 internações hospitalares por causas sensíveis à atenção básica ${ }^{1}$ na Bahia, considerando hospitais públicos e privados e todas as categorias de atendimento. Dessas $n=9.677(1,65 \%)$ foram relacionados a diabetes mellitus, $n=53(0,009 \%)$ à obesidade e $n=5.735$ (0,97\%) à hipertensão (Tabela 1). A Tabela 1 ainda apresenta as características sociodemográ- ficas dos usuários internados por DM, obesidade e HAS, na Bahia e em Salvador.

Em relação a Salvador, o número de internações registradas foi de 170.176 , sendo, $0,63 \%(n=1.079)$ por diabetes mellitus, $0,03 \%(n=52)$ por obesidade e $0,85 \%$ $(n=1.451)$ por hipertensão. Dentre as internações ocorridas por DM, verificou-se maior prevalência de homens, $52,27 \%$ ( $n=564$ ), idosos, $56,81 \%$ ( $n=613$ ), sem informação de cor ou raça, $50,50 \%(n=545)$, seguidos de pardos $34,20 \%(n=369)$. Quando observadas as características das internações relacionadas à obesidade, tem-se uma prevalência de mulheres, $90,38 \%$ ( $n=47)$, de adultos, 92,31 $(n=48)$ e sem informação de cor ou raça, $82,69 \%(n=43)$, seguidos de branca e amarela, ambas com $5,77 \%(n=3)$. Por último, nas internações por HAS, percebeu-se maior prevalência de mulheres, $82,08 \%(n=1.191)$, de idosos, $85,32 \%$ ( $n=1.238)$, e da cor ou raça autodeclarada preta, $43,97 \%(n=638)$ (Tabela 1).

Tabela 1 - Frequência de internamentos e características sociodemográficas de usuários, segundo lista de morbidade hospitalar (CID10), nos meses de janeiro a novembro de 2019, na Bahia e em Salvador.

\begin{tabular}{|c|c|c|c|c|c|c|}
\hline \multirow[t]{3}{*}{ Variáveis } & \multicolumn{2}{|c|}{ Diabetes mellitus } & \multicolumn{2}{|c|}{ Obesidade } & \multicolumn{2}{|c|}{ Hipertensão essencial } \\
\hline & Salvador & Bahia & Salvador & Bahia & Salvador & Bahia \\
\hline & n (\%) & n (\%) & $\mathrm{n}(\%)$ & n (\%) & $\mathrm{n}(\%)$ & n (\%) \\
\hline \multicolumn{7}{|l|}{ Sexo } \\
\hline Masculino & $564(52,27)$ & $4.674(48,30)$ & $5(9,61)$ & $5(9,43)$ & $260(17,92)$ & $2.062(35,95)$ \\
\hline Feminino & $515(47,73)$ & $5.003(51,7)$ & $47(90,38)$ & $48(90,57)$ & $1.191(82,08)$ & $3.673(64,05)$ \\
\hline Total & 1.079 & 9.677 & 52 & 53 & 1.451 & 5.735 \\
\hline \multicolumn{7}{|l|}{ Faixa etária } \\
\hline 20 a 59 anos & $466(43,19)$ & $3.773(38,99)$ & $48(92,31)$ & $49(92,45)$ & $213(14,68)$ & $2.044(35,64)$ \\
\hline 60 anos ou mais & $613(56,81)$ & $5.904(61,01)$ & $4(7,69)$ & $4(7,55)$ & $1.238(85,32)$ & $3.691(64,36)$ \\
\hline Total & 1.079 & 9.677 & 52 & 53 & 1.451 & 5.735 \\
\hline \multicolumn{7}{|l|}{ Raça ou cor* } \\
\hline Branca & $22(2,04)$ & $497(5,14)$ & $3(5,77)$ & $3(5,66)$ & $233(16.06)$ & $473(8,25)$ \\
\hline Preta & $92(8,53)$ & $513(5,30)$ & $1(1,92)$ & $1(1,89)$ & $638(43,97)$ & $864(15,06)$ \\
\hline Parda & $369(34,20)$ & $5.777(59,70)$ & $2(3,85)$ & $3(5,66)$ & $287(19,78)$ & $3.268(56,98)$ \\
\hline Amarela & $51(4,73)$ & $338(3,49)$ & $3(5,77)$ & $3(5,66)$ & $25(1,72)$ & $192(3,35)$ \\
\hline Indígena & - & $3(0,03)$ & - & - & - & $1(0,02)$ \\
\hline Sem informação & $545(50,50)$ & $2.549(26,34)$ & $43(82,69)$ & $43(81,13)$ & $268(18,47)$ & $937(16,34)$ \\
\hline Total & 1.079 & 9.677 & 52 & 53 & 1.451 & 5.735 \\
\hline
\end{tabular}

Fonte: Ministério da Saúde - Sistema de Informações Hospitalares do SUS (SIH/SUS).

*Cor ou raça autodeclarada. **Dos casos de internação por obesidade ocorridos na Bahia, um foi registrado no município de São Felix e os outros foram registrados em Salvador. CID-10 - Classificação Estatística Internacional de Doenças e Problemas Relacionados à Saúde, versão 10.

A taxa de mortalidade [razão entre a quantidade de óbitos e o número de Autorização por Internações Hospitalares (AIH) pagas, computadas como internações, no período, multiplicada por 100], em Salvador, foi de 5,93 e 3,1 para DM e HAS, respectivamente. Essas taxas foram maiores que as do Nordeste e do Brasil e em relação à HAS, maior que a da Bahia (Tabela 2).

1 As Condições Sensíveis à Atenção Primária estão listadas por grupos de causas de internações e diagnósticos, de acordo com a Décima Revisão da Classificação Internacional de Doenças (CID-10) - PORTARIA MS № 221, DE 17 DE ABRIL DE 2008. 
Tabela 2 - Taxa de mortalidade por diabetes mellitus, obesidade e hipertensão essencial, considerando-se os meses de janeiro e novembro de 2019.

\begin{tabular}{lcccc}
\hline & Brasil & Nordeste & Bahia & Salvador \\
\hline Diabetes mellitus & 4,51 & 4,81 & 7,01 & 5,93 \\
Obesidade & 0,22 & 0,20 & - & - \\
Hipertensão essencial & 1,63 & 1,91 & 2,88 & 3,1 \\
\hline
\end{tabular}

Fonte: Ministério da Saúde - Sistema de Informações Hospitalares do SUS (SIH/SUS)

Óbitos por todas as causas sensíveis à atenção básica [conjunto de problemas de saúde para os quais a efetiva ação da atenção primária diminuiria o risco de internações (ALFRADIQUE et al., 2006)], registrados de janeiro a novembro de 2019, na Bahia, perfizeram um total de 27.086. Em Salvador, esse número foi de 7.940. Dos óbitos ocorridos em Salvador, $0,80 \%(n=64)$ foram relacionados à DM e $0,56 \%(n=45)$ relacionados à HAS. Nota-se que a totalidade dos óbitos por obesidade registrados no Nordeste foram cadastrados no estado de Pernambuco. A Tabela 3 mostra os óbitos, por lista de morbidade, ocorridos no Nordeste, na Bahia e em Salvador.
Tabela 3 -Óbitos por diabetes mellitus, obesidade e hipertensão essencial, nos meses de janeiro a novembro de 2019.

\begin{tabular}{lccc}
\hline & Nordeste & Bahia & Salvador \\
\hline Diabetes mellitus & 1.766 & 678 & 64 \\
Obesidade & 2 & - & - \\
Hipertensão essencial & 332 & 165 & 45 \\
\hline
\end{tabular}

Fonte: Ministério da Saúde - Sistema de Informações Hospitalares do SUS (SIH/SUS).

*Não foram registrados óbitos por obesidade na Bahia, nesse período.

Dos óbitos por DM, 51,56\% foram de pacientes do sexo feminino, $73,44 \%$ de idosos, e $50 \%$ sem informação de cor ou raça autodeclarada, e $32,81 \%$ de cor parda. Quando observados os casos de HAS, verificou-se maior prevalência do sexo feminino, $68,86 \%$, de idosos, $82,22 \%$, e sem informação de cor ou raça autodeclarada, $48,89 \%$, seguida de cor parda, 22,22\% (Tabela 4).

Tabela 4 - Óbitos segundo lista de morbidade hospitalar CID10, nos meses de janeiro a novembro de 2019. Salvador, Bahia.

\begin{tabular}{|c|c|c|c|c|}
\hline \multirow[t]{3}{*}{ Variáveis } & \multicolumn{2}{|c|}{ Diabetes mellitus } & \multicolumn{2}{|c|}{ Hipertensão essencial } \\
\hline & Salvador & Bahia & Salvador & Bahia \\
\hline & n (\%) & n (\%) & n (\%) & n (\%) \\
\hline \multicolumn{5}{|l|}{ Sexo } \\
\hline Masculino & $31(48,44)$ & $318(46,90)$ & $14(31,11)$ & $71(43,03)$ \\
\hline Feminino & $33(51,56)$ & $360(53,10)$ & $31(68,89)$ & $94(56,97)$ \\
\hline Total & 64 & 678 & 45 & 165 \\
\hline \multicolumn{5}{|l|}{ Faixa etária } \\
\hline 20 a 59 anos & $17(26,56)$ & $167(24,63)$ & $8(17,78)$ & $34(20,61)$ \\
\hline 60 anos ou mais & $47(73,44)$ & $511(75,37)$ & $36(82,22)$ & $131(79,39)$ \\
\hline Total & 64 & 678 & 45 & 165 \\
\hline \multicolumn{5}{|l|}{ Raça ou Cor* } \\
\hline Branca & - & $29(4,28)$ & $4(8,89)$ & $12(7,27)$ \\
\hline Preta & $9(14,06)$ & $44(6,49)$ & $6(13,33)$ & $12(7,27)$ \\
\hline Parda & $21(32,81)$ & $408(60,18)$ & $10(22,22)$ & $89(53,94)$ \\
\hline Amarela & $2(3,13)$ & $21(3,09)$ & $3(6,67)$ & $6(3,64)$ \\
\hline Indígena & - & - & - & - \\
\hline Sem informação & $32(50)$ & $176(25,96)$ & $22(48,89)$ & $46(27,88)$ \\
\hline Total & 64 & 678 & 45 & 165 \\
\hline
\end{tabular}

Fonte: Ministério da Saúde - Sistema de Informações Hospitalares do SUS (SIH/SUS).

*Cor ou raça autodeclarada. **Não foram registrados óbitos por obesidade na Bahia nesse período.

CID-10 - Classificação Estatística Internacional de Doenças e Problemas Relacionados à Saúde, versão 10.

\section{DISCUSSÃO}

A HAS e o DM configuram graves problemas de saúde no Brasil, devido a suas elevadas prevalências e complicações agudas e crônicas. O cenário da HAS e do DM tem sido agravado pela transição demográfica e nutricional acelerada, sendo os idosos o grupo de maior risco, justificando a predominância desse grupo etário, nas internações por DM e HAS, no presente estudo (GERHARDT et al., 2016).

A HAS e o DM são doenças comuns ao envelhecimento, e seu controle tem se tornado um desafio para o Sistema Público de Saúde. Segundo Carreira et al (2016), 
estudos estimam que a prevalência mundial de HAS seja de um bilhão de indivíduos, provocando, aproximadamente, 7,1 milhões de mortes todos os anos. No Brasil, sua prevalência varia de $22 \%$ a $44 \%$ para adultos, chegando a mais de $50 \%$ para idosos de 60 a 69 anos e $75 \%$ em idosos com mais de 70 anos (GERHARDT et al., 2016). A HAS é considerada um grande problema mundial de saúde pública, sendo a condição clínica mais comumente encontrada na atenção primária à saúde, responsável por, aproximadamente, 9,4 milhões de mortes por ano no mundo (SANTIAGO et al., 2019).

Outro importante aspecto relacionado à HAS é a composição corporal, especialmente no que tange à distribuição da adiposidade, estando claro que o aumento do tecido adiposo visceral está diretamente associado a uma maior incidência da doença (SANTIAGO et al., 2019). $O$ excesso de peso (sobrepeso e de obesidade) constitui o sexto fator de risco mais importante para a carga global de doenças, em face de sua associação com várias DCNT, incluindo doenças cardiovasculares, como a hipertensão arterial e acidente vascular cerebral, diabetes, câncer de cólon, reto e de mama, cirrose, gota, osteoartrite e apneia do sono (MALTA et al., 2014). Nesta pesquisa, não ficou claro, no banco de dados do DATASUS, se os usuários apresentavam mais de uma comorbidade, o que agravaria o risco de morte e o aumento do número de óbitos.

Quando observadas, neste estudo, as internações por DM, verificou-se que o grupo de maior predominância foi o de idosos, situação semelhante à dos resultados encontrados em um estudo realizado no estado do Ceará, no período de 2001 a 2012, com indivíduos adultos e idosos. Esse quadro pode ser justificado pelas alterações fisiológicas relacionadas ao processo de envelhecimento, ou ainda pode ser reflexo das complicações decorrentes da duração da doença (GERHARDT et al., 2016).

Ao observar as internações por obesidade na Bahia e em Salvador, observa-se uma pequena diferença nesses números e questiona-se a representatividade deles nesses casos, considerando a quantidade de municípios que compõem o estado e o padrão etiológico que acompanha os agravos pesquisados. Acredita-se que tal prevalência não corresponda à realidade, podendo ser subestimada por deficiência na alimentação do SIH/SUS, falha na implementação do sistema, ou dificuldade no acesso às unidades de saúde, por indivíduos acometidos por obesidade. Conforme Cascão et al. (2016), não há regras explícitas para a emissão e preenchimento dos dados da $\mathrm{AlH}$, tampouco treinamento padronizado e regular das equipes. Por isso, a qualidade da informação de prontuários médicos e de codificação do diagnóstico principal também afetam a qualidade dos dados do $\mathrm{SIH}$ -SUS (CASCÃO et al., 2016). Vale ressaltar que, de acordo com informações do DATASUS, a causa da internação é a informada como diagnóstico principal, ou seja, aquela que motivou a internação, sendo diretamente transcrita do prontuário médico; é de responsabilidade do serviço de registro das unidades prestadoras. Como a obesidade pode ser o fator etiológico para o desenvolvimento de outras doenças, talvez a presença de enfermidades com risco de morte seja o relatado no prontuário e não aquela.

De modo geral, este estudo mostrou que, na maioria das doenças pesquisadas, apesar da maior incidência de casos no sexo masculino, mulheres se internaram mais que homens, sendo um resultado esperado, considerando que mulheres tendem a cuidar da saúde e manter os níveis pressóricos mais controlados que os homens. As mulheres são mais presentes nos serviços de saúde e continuam o tratamento mais que os homens. Por uma questão cultural, os homens não têm o hábito de buscar assistência e de cuidar da saúde (DANTAS et al., 2018). Por outro lado, sob outra perspectiva, por frequentarem mais os serviços de saúde, possuem maior risco de internamento, e os homens que protelam essa busca poderiam ter menores taxas de internação.

Em relação às características sociodemográficas, o grupo etário mais prevalente, nas internações por HAS e DM, foi o maior de 60 anos, achado semelhante ao do estudo de Dantas et al. (2018), resultado esperado em função do efeito deletério da HAS e DM no organismo, decorrente do processo de envelhecimento. Os idosos, em função da maior carga de doenças e incapacidades, utilizam grande parcela dos serviços de saúde, refletindo a ineficiência dos modelos de atenção vigentes para eles. Considerando a cor ou raça autodeclarada, verificou-se grande prevalência de ausência de informações, com referência a todas as doenças pesquisadas, fato semelhante ao ocorrido no estudo de Dantas et al. (2018), e isso pode comprometer o planejamento e a alocação de recursos para ações voltadas para a real necessidade em saúde (DANTAS et al., 2018). Essa falta de informação pode estar relacionada à dificuldade de quem entrevista em solicitar informações sobre esse dado, ou por não ter clareza da importância dele, ou ainda da dificuldade de autodeclarar cor ou raça, por parte do usuário, devido ao preconceito ou à falta de conhecimento. Pode também estar relacionada às características negativas atreladas à classificação autorreferida de "não branco", especialmente atribuída aos negros (ARAÚJO, 2016).

Vale ressaltar que, segundo Malta et al. (2017), a prevalência de HAS chega a ser até $130 \%$ maior entre as mulheres pretas, quando comparadas às brancas. Isso se relaciona a fatores como predisposição genética, piores condições de vida, menor acesso aos serviços de saúde e estresse devido à discriminação racial. Esse dado é de grande relevância considerando a composição étnico-racial da população soteropolitana (MALTA et al., 2017).

Quando observados os óbitos por HAS e DM, verificou-se maior prevalência de mulheres, dado semeIhante ao do estudo de Coelho et al. (2019), no qual o quantitativo de pessoas falecidas com hipertensão era maior entre as do sexo feminino. Nesse mesmo estudo, das doenças associadas a fatores de risco cardiovascular, que tiveram prevalência relevante na população estudada, apenas a presença de DM se associou à hipertensão no 
modelo multivariado (COELHO et al., 2019). Em outro estudo, autores advertem que a região Nordeste do Brasil é detentora de maior incidência de pobreza, e seus habitantes apresentam duas vezes mais chances de evoluir a óbito durante uma hospitalização por DM. Isso se deve à insatisfação, entre usuários e profissionais de saúde, a respeito do tratamento (insuficiente) fornecido pela Atenção Primária à Saúde (APS), que pode influenciar no aumento da morbidade hospitalar (SOUZA JUNIOR et al., 2019). Apesar de o presente estudo não apresentar prevalência de óbitos por obesidade na Bahia, estimativas para 2020 apontam cerca de cinco milhões de óbitos atribuídos ao excesso de peso, no mundo (MALTA et al. 2014).

Neste estudo, ainda se observa que as taxas de mortalidade do Nordeste se apresentaram muito semelhantes às do Brasil, evidenciando o alto risco das DCNT. Segundo Medeiros et al. (2018), as DCNT constituem a causa de $80 \%$ dos óbitos que ocorrem em países de baixa renda, devido a uma maior exposição a fatores de risco e menor acesso aos serviços de saúde, quando comparados aos países de alta renda per capita. Além disso, as diferenças existentes entre regiões, na qualidade da assistência prestada, na capacidade diagnóstica e na qualidade das informações fornecidas também são importantes para reduzir as taxas de mortalidade, intensificar o controle dos fatores de risco e favorecer o acesso aos serviços de saúde (MEDEIROS et al., 2018).

A ocorrência de internações gera custos diretos à saúde, pela utilização dos recursos clínicos, pelas despesas com doentes e familiares, com deslocamentos para a assistência; e indiretos, relacionados à perda de função e da produtividade, decorrentes dos problemas de saúde (DANTAS et al., 2018). A análise das internações por doenças, como o DM, a obesidade e a HAS, fornece-nos um panorama do comportamento dessas doenças, permitindo seu monitoramento, além de ser uma forma de acompanhar a efetividade das ações na APS, já que esse nível de atenção é responsável pelos cuidados primários e pela detecção da doença, evitando complicações e a necessidade de internação. Sendo assim, as hospitalizações tornam-se um indicador da qualidade da assistência prestada pela APS, que, quando feita corretamente, leva à redução das internações por condições sensíveis (hospitalizações potencialmente evitáveis) a essa assistência (GERHARDT et al. 2016).

\section{CONCLUSÃO}

Neste estudo, foi observada maior prevalência de mulheres e idosos nas internações e óbitos por HAS e DM, apesar da multiplicidade dos dados encontrados. Esses resultados mostram que principalmente a prevenção, além do acompanhamento e controle do agravamento das DCNT pelos serviços de saúde são de extrema importância em Salvador (BA), reforçando a importância das APS nessa relação. Deve-se considerar a malignidade dessas comorbidades e suas consequências não apenas para os cofres públicos, mas para a sociedade como um todo.
Vale ressaltar que o uso dos dados do DATASUS foi de extrema importância para entender o perfil de morbidade hospitalar. No entanto, é necessária uma padronização no modo de coleta desses dados, a fim de garantir sua consistência e qualidade.

\section{REFERÊNCIAS}

ALFRADIQUE, M. E. et al. Internações por condições sensíveis à atenção primária: a construção da lista brasileira como ferramenta para medir o desempenho do sistema de saúde (Projeto ICSAP - Brasil). Cad. Saúde Pública, Rio de Janeiro, v.25, n.6, p. 1337-1349, jun. 2009

ARAÚJO, E. M. Q. Intervenção dietoterápica na síndrome metabólica e sua associação com o perfil genético da intolerância à lactose. 2016. Tese (Doutorado em Biotecnologia) - Feira de Santana: Universidade Estadual de Feira de Santana, Salvador, 2016.

ARAÚJO, F. K. de et al. Atenção Nutricional para Obesidade em Unidades Básicas de Saúde. Revista Brasileira de Obesidade, Nutrição e Emagrecimento, São Paulo, v. 13. n. 79. p.385-393, maio/jun. 2019. ISSN 1981 - 9919

ASSOCIAÇÃO BRASILEIRA PARA O ESTUDO DA OBESIDADE E DA SÍNDROME METABÓLICA(ABESO). Diretrizes brasileiras de obesidade. 2016. 4.ed. São Paulo: ABESO, 2016.

BRASIL. Ministério da Saúde. Portaria no 221, de 17 de Abril de 2008. Lista Brasileira de Internações por Condições Sensíveis à Atenção Primária. Diário Oficial [da] República Federativa do Brasil, Brasília, DF, 2008. Disponível em: http://bvsms.saude.gov.br/bvs/saudelegis/sas/2008/ prt0221_17_04_2008.html. Acesso| em: 12 mar. 2020.

BRASIL. Ministério da Saúde. Departamento de Informática do SUS(DATASUS). Informações de Saúde TABNET. Brasília: DATASUS, 2008. Disponível em: http://www2.datasus.gov.br/DATASUS/index. php?area=02. Acesso em: 02 set. 2020.

CASCÃO, A. M. et al. Uso do diagnóstico principal das internações do Sistema Único de Saúde para qualificar a informação sobre causa básica de mortes naturais em idosos. Rev. Bras. Epidemiol., São Paulo, v.19, n.4, p. 713-726, out./dez. 2016.

COELHO, J. C. et al. Hypertension is the underlying cause of death assessed at the autopsy of individuals. Rev. Esc. Enferm. USP, São Paulo, v.53, p. e03457, 2019. DOI: 10.1590/S1980-220X2018006103457

DANTAS, R. C. de et al. Fatores associados às internações por hipertensão arterial. Einstein (São Paulo), São Paulo, v.16, n.3, p. eAO4283, 2018. DOI: 10.1590/ S1679-45082018AO4283.

DIAS, P. C. et al. Obesidade e políticas públicas: concepções e estratégias adotadas pelo governo brasileiro. Cad. Saúde Pública, Rio de Janeiro, v.33, n.7, p.e00006016, 2017.

DUTRA, M. T. et al. Obesidade e Hipertensão no Distrito Federal, Brasil. Rev. Aten. Saúde. Rev. Aten. Saúde, São Paulo, v. 17, n.59, p. 4-9, 2019.

FERREIRA, A. P. S.; SZWARCWALD, C. L.; DAMACENA, G. N. Prevalência e fatores associados da obesidade na população brasileira: estudo com dados aferidos da Pesquisa Nacional de Saúde, 2013. Rev. Bras. Epidemiol., São Paulo, v.22, n. E190024, 2019.

FLOR, L. S.; CAMPOS, M. R. Prevalência de diabetes mellitus e fatores associados na população adulta brasileira: evidências de um inquérito de base populacional. Rev. Bras. Epidemiol., São Paulo, v.20, n.1, p. 16-29, jan./mar. 2017.

FRANCISCO, P. M. S. B. et al. Prevalência simultânea de hipertensão e diabetes em idosos brasileiros: desigualdades individuais e contextuais. Ciênc. Saúde Colet., v.23, n.11, p.3829-3840, 2018. 
GERHARDT, P. C. et al. Tendência das internações por diabetes mellitus e hipertensão arterial sistêmica em idosos Cogitare Enferm., Curitiba, v. 21, n.4, p. 01-10, out./dez. 2016.

MALTA, D. C. et al. Evolução anual da prevalência de excesso de peso e obesidade em adultos nas capitais dos 26 estados brasileiros e no distrito federal entre 2006 e 2012. Rev. Bras. Epidemiol., São Paulo p. 267-276, 2014.

MALTA, D. C. et al. Prevalência e fatores associados com hipertensão arterial autorreferida em adultos brasileiros. Rev. Saúde Publica, São Paulo, v.51, supl. 1, p. 11s, 2017.

MEDEIROS, J. dos et al. Mortalidade por infarto agudo do miocárdio. Rev. Enferm. UFPE online., Recife, v.12, n.2, p. 565-572, fev. 2018

MINISTÉRIO DA SAÚDE. Secretaria de Vigilância em Saúde. Boletim Epidemiológico. v. 50, n. 40. Dez. 2019

SANTIAGO, E. R. C. et al. Prevalência e Fatores Associados à Hipertensão
Arterial Sistêmica em Adultos do Sertão de Pernambuco, Brasil. Arq. Bras Cardiol., São Paulo, v.113, n.4, p.687-695, 2019.

SOCIEDADE BRASILEIRA DE CARDIOLOGIA (SBC). SOCIEDADE BRASILEIRA DE HIPERTENSÃO (SBH). SOCIEDADE BRASILEIRA DE NEFROLOGIA (SBN). 7a Diretriz Brasileira de Hipertensão Arterial. Rev. Bras. Hipertens., Rio de Janeiro, v.24, n.1, p. 12-7, 2017.

SOCIEDADE BRASILEIRA DE DIABETES (SBD). Diretrizes da Sociedade Brasileira de Diabetes 2019-2020. São Paulo: Editora Clannad, 2019.

SOUZA JÚNIOR, E. V. de et al. Internações, óbitos e custos hospitalares por diabetes mellitus. Rev. Enferm. UFPE online., Recife, v.13, p.e240388, 2019. DOI: 10.5205/1981-8963.2019.240388.

SWINBURN, B. A. et al. The lancet commissions the global syndemic of obesity, undernutrition and climate change. Lancet Commissions, [s.I], v.393, n. 10173, 2019.

Submetido em: 02/11/2020

Aceito em: 05/11/2020 